\title{
La Révolution française. Idéaux, singularités
}

\section{Élizabeth Liris}

\section{OpenEdition}

Journals

Édition électronique

URL : https://journals.openedition.org/ahrf/2221

DOI : 10.4000/ahrf.2221

ISSN : $1952-403 X$

Éditeur :

Armand Colin, Société des études robespierristes

Édition imprimée

Date de publication : 1 septembre 2005

Pagination : 185-188

ISSN : 0003-4436

\section{Référence électronique}

Élizabeth Liris, «La Révolution française. Idéaux, singularités », Annales historiques de la Révolution

française [En ligne], 341 | juillet-septembre 2005, mis en ligne le 27 avril 2006, consulté le 22 avril 2022. URL : http://journals.openedition.org/ahrf/2221 ; DOI : https://doi.org/10.4000/ahrf.2221

Ce document a été généré automatiquement le 22 avril 2022.

Tous droits réservés 


\title{
La Révolution française. Idéaux, singularités
}

\author{
Élizabeth Liris
}

\section{RÉFÉRENCE}

Robert Chagny, dir., La Révolution française. Idéaux, singularités, influences. Actes des journées d'études en hommage à Albert Soboul, Jacques Godechot et Jean-René Suratteau, Grenoble, Presses Universitaires de Grenoble, 2002, 410 p., ISBN 2-7061-1058-9, 22 e.

1 Les journées d'études en hommage à ces grands historiens de la Révolution s'orientent autour de trois grands axes: la nation, la frontière et l'expansion de la Révolution française, qui intègre le thème de la révolution occidentale, largement étudié par ces auteurs dans les années cinquante.

2 La première partie s'attache à repenser la notion de "révolution atlantique » et le concept de "Grande Nation ». Dans le contexte géopolitique des années 1950, Jacques Godechot et Robert R. Palmer se voient accusés d'être au service de l'OTAN, en pensant une révolution atlantique qui s'étirerait dans la longue durée, de 1770 à 1848, de la révolution en Amérique du Nord aux révolutions de 1848, en un temps où la Révolution française en tant que modèle unique et de référence obligée se serait imposée. Wolfgang Schmale montre bien comment les notions catégorielles se modifient et l'idée de révolution atlantique se transforme en concept d'histoire transatlantique s'étendant à l'ouest de l'Afrique, à l'est de l'Amérique du Nord et du sud, aux Caraïbes et à l'Europe atlantique. L'histoire atlantique privilégie les dynamismes des relations réciproques migrations, volontaires ou involontaires, esclavage - et aboutit à la naissance «d'un nouveau soi culturel de l'autre côté de l'Atlantique »; Donald William Meinig parle des "prescriptions réciproques du soi-européen et du soi-nord-américain». Les recherches pourraient être poursuivies sur ces échanges et ces transferts culturels, ainsi que sur l'émergence d'une société civile transnationale. 
3 Abordant le temps de la Révolution, Martine Acerra montre la supériorité de la Royal Navy sur la flotte de guerre française, en dépit des efforts de la Royale, comme en témoigne la bataille d'Aboukir. Pourtant, on constate qu'en Bretagne, des ports comme Saint-Malo, Nantes et Lorient ont connu une réelle embellie au $\mathrm{xVIII}^{\mathrm{e}}$ siècle; leur prospérité est due en partie, comme le montre Silvia Marzagalli, au trafic triangulaire et au commerce avec les îles. Pendant la décennie révolutionnaire, les guerres vont profondément toucher certaines régions de Bretagne; aux pertes humaines vont s'ajouter des difficultés économiques parfois considérables pour les armateurs, les négociants ou manufacturiers, les pêcheurs et les paysans. Partout les relations commerciales se modifient, comme en témoigne la compétition entre Hambourg, ville impériale et Bordeaux. La situation devient encore plus complexe avec la naissance « de nouvelles formes de colonialisme, non pour la conquête de terres mais pour celle des marchés ». Le système colonial semble avoir vécu et "une révolution du commerce atlantique s'est opérée ». La « dialectique de l'ouverture et du repli» (Éric Wauters) se marque en Normandie, avec la création en 1792 de la Société d'Émulation de Rouen pour l'encouragement de l'agriculture, de la pêche, des manufactures, des arts et du commerce, et on constate la continentalisation de Rouen avec son éloignement des activités maritimes, voire son hostilité au colonialisme, parfois lié à l'anglophobie ; les structures sociales s'en trouvent donc modifiées. Pour clore cette première séquence, Marcel Dorigny, dans un bilan historiographique, se fait le porte-parole des récents travaux sur la question coloniale, l'esclavage, le monde des savants et les réseaux transatlantiques (dont certains restent pro-esclavagistes). Le mouvement abolitionniste et le rôle de la Société des Amis des Noirs ont été revus, ainsi que la datation des ruptures essentielles qui doit se placer en l'an VIII plutôt qu'en l'an III (voir les colloques publiés en 1995 et 1999, à Paris, sous la direction de M. Dorigny), ou encore la question du projet colonial dans la longue durée. Revisiter cette question amène donc à considérer l'ambiguïté du message des Lumières, largement prolongé par les révolutionnaires et qui, hélas « sera porteu[r] du projet colonial ».

La seconde séquence porte sur la Révolution française et l'éclosion des nationalités dans les espaces italien, germanique et helvétique. Pour J.-R. Suratteau, le principe des nationalités reposait sur des bases juridiques, naturelles, culturelles, économiques et sociales, liées au processus de développement historique dans lequel il s'inscrivait. Si les Lumières opposent l'esprit national à l'universalité, la Révolution française contribue à l'éclosion des nationalités (Georges Andrey) ; Philippe Gut souligne qu'en Allemagne, la Heimat, petite patrie, correspondait au pays où on était né, où on avait vécu, où on mourrait ; avec l'émergence d'un sentiment national, la langue contribue à l'unification de la nation. Dans l'espace helvétique, le sentiment patriotique s'éveille aussi avant la Révolution, mais les difficultés dues aux différences de langue et de religion, ainsi que les antagonismes sociaux nuisent à la formation d'un sentiment national «entre cantons dominateurs et pays assujettis»; finalement, les progrès seront « insuffisants pour constituer les bases d'une authentique unité nationale ». Si l'Italie, divisée entre de nombreuses entités politiques parfois rivales désire l'unité économique, elle ne se montre pas prête à accepter des réformes sociales. C'est donc plutôt l'occupation étrangère qui sera le ferment de l'unité avant la Révolution. L'impact de la Révolution et le "mythe de la Grande Nation» se marquent chez les patriotes italiens par l'espoir d'une unité, puis se retourne contre l'occupant pour devenir anti-français en 1799. En Allemagne, enfin, la volonté de former une nation prendra force avec le « discours à la nation allemande » de Fichte, après la formation de 
la Confédération germanique. On peut donc dire que le message révolutionnaire est un des catalyseurs des mouvements nationaux dans ces trois territoires, mais que le sentiment patriotique finit par se fortifier contre son propre instigateur.

C'est ce que démontre Marita Gilli, qui rappelle le rôle de la bourgeoisie éclairée allemande, mais aussi l'importance des sentiments de cosmopolitisme et de xénophobie dans ces territoires. Elle évoque aussi la pensée de Herder rejetant le modèle français, mais privilégiant les références antiques et réhabilitant le Moyen Âge, sans remettre en cause le despotisme de Frédéric II ; le patriotisme est devenu « une valeur politique subversive ». Le patriotisme s'incarne dans l'idée de créer une communauté de citoyens libres, dans un État uni, mais avec l'occupation française vient le temps du rejet puis de l'hostilité contre l'usurpateur. C'est en Italie que Gilles Bertrand retrouve «le cosmopolitisme à l'épreuve de la Révolution française ", en interrogeant les émigrés, nombreux à Turin dès 1789 , puis à Rome et à Véronne (les prêtres réfractaires se regroupent surtout dans les États Pontificaux). L'Italie, autrefois destination de voyages et de loisirs pour ces aristocrates argentés devient, avec la Révolution, une terre d'exil, une rupture, comme en témoignent leurs correspondances montrant un cosmopolitisme peu à peu " piraté » qui, finalement, réactive l'amour de leur ancienne patrie. Dans la même optique, à partir de la correspondance du consul français à Tunis, Rachida Tlili Sellaouti étudie le concept de «Grande Nation dans l'espace méditerranéen "; elle en isole une évolution sémantique, car la notion, tout en ne s'appliquant qu'aux faits militaires représente un véritable espace de liberté et de libération (pour les captifs étrangers) « dans cet environnement barbaresque ». Mais, à partir de 1801, l'emploi de l'expression disparaît. Si la Grande Nation a dans un premier temps crée un espace de liberté en Méditerranée, à long terme, elle n'a pas pu former un espace juridique incluant la Régence de Tunis et les autres Régences barbaresques.

Revenant sur l'espace helvétique, Georges Andrey, Hubert Forest et Alain-Jacques Czouz-Tornare se penchent sur plusieurs points précis. G. Andrey tente de cerner le jacobin suisse entre mythe et réalité de 1798 à 1805 . Reprenant la démarche de J. Godechot - qui a, le premier, élargi la qualification de jacobin - et l'étude de Michel Vovelle sur Les Jacobins de Robespierre à Chevènement (1999), il présente le jacobin suisse comme un modéré. À partir de textes contextualisés et de l'exemple d'un jacobin avoué, Jean-Jacques Cart (1748-1813), le jacobin helvétique apparaît comme un honnête citoyen, démocrate, exerçant le droit de vote, mais aussi anti-aristocrate, admirateur de la France nouvelle - « celle de la liberté, de l'égalité, de la fraternité »-; la question reste pourtant l'un des chantiers à revisiter de façon générale pour toutes les républiques sœurs. L'exposé de $\mathrm{H}$. Foerster, pour sa part, s'intéresse à "l'opposition populaire à la République helvétique, 1798-1813». La Suisse doit faire face à une opposition civile et militaire qui reste toujours divisée : en 1799, des soulèvements contre-révolutionnaires se forment; en 1802, des luttes politiques voient s'affronter républicains et unitariens.

7 Florence Catherine, quant à elle, analyse les liens entre académies scientifiques allemandes et françaises en suivant l'itinéraire de l'astronome Roger Barry et du marquis Charles de Villers à Mannheim et à Göttingen en liaison avec Paris. La Révolution jusqu'en 1792 prolonge les liens tissés à la fin de l'Ancien Régime, et ces contacts perdurent très largement jusqu'en 1815 - à considérer l'exemple du marquis de Villers -; ces contacts autorisent «de nouvelles relations intellectuelles francogermaniques revivifiées et réorientées par une nouvelle indépendance intellectuelle 
allemande qui explosa littéralement dans les dernières années de l'empire napoléonien ". L'influence française se retrouve, dans un autre registre, celui des écrivains contre-révolutionnaires (1795-1800). En s'appuyant sur les écrits de Burke, Joseph de Maistre, Mallet du Pan, Francis d'Ivernois Calonne ou Gentz, Marc Belissa interroge ces hommes hostiles à la Révolution, conscients de la faillite de l'Ancien Régime (qu'ils continuent cependant d'idéaliser), mais en quête d'un nouveau modèle la Révolution appartient au domaine du non droit - et d'une stabilisation de l'ordre européen que la Révolution n'a fondé que sur la violence, tout en cherchant une éventuelle coexistence des Républiques et des monarchies (voir les travaux d'A. M. Rao pour l'Italie ). Pour conclure cette seconde séquence, Annie Jourdan se propose d'aborder les idéaux, singularités et influences de la Révolution française en GrandeBretagne, aux États-Unis et dans les Provinces-Unies, en nuançant les conclusions de J. Godechot et de R. Palmer qui ont porté l'accent plus sur l'unité que sur la diversité. Aux modèles et aux extrapolations hâtives d'une histoire plus récente, l'auteur préfère une lecture dans la longue durée, sur les aspects politiques mais aussi sociaux de ces révolutions occidentales; elle insiste sur le fait qu'en dépit de situations initiales, de conjonctures et de méthodes très différentes «elles manient les mêmes références républicaines; elles connaissent des modes d'organisation et de mobilisations identiques et donc une intense sociabilité politique, elles fondent des constitutions écrites ".

8 La troisième séquence de ces journées d'études se consacre, d'une part, aux pratiques politiques et aux élections, et, d'autre part, aux rapports entre libéralisme et démocratie. Depuis le Bicentenaire, Bernard Gainot et Serge Aberdam en collaboration avec de nombreux chercheurs (atlas de Jean Boutier et Philippe Boutry) explorent avec minutie ce terrain longtemps resté en friches par les spécialistes. Quatre communications s'attachent aux pratiques politiques et aux systèmes électoraux. Danièle Pingué se penche sur deux régions : une Haute-Normandie plutôt engagée et une Franche- Comté plutôt hostile à la Révolution, ce qui influe, comme le montrent les cartes, de façon très variable sur les pratiques électorales. Dans la Lorraine rurale (1790-1800) l'apprentissage de la démocratie est abordé par Jean-Paul Rothiot, qui analyse 400 élections municipales et 100 élections primaires dans quinze cantons et cent vingt-trois communes, ce qui a permis de construire une précieuse base de données; l'auteur souligne le légalisme républicain qui semble inciter les habitants à accepter tous les changements, au nom de l'attachement à la République, honorée par l'assistance aux fêtes, comme par la pratique du serment. Ce dernier est au cœur des comportements religieux entre 1791 et 1801. Malcolm Crook aborde les élections ecclésiastiques, assez peu étudiées; la pratique est non seulement nouvelle, mais également délicate à cause de la division entre constitutionnels et réfractaires - ces derniers refusent de participer aux élections -. Dans l'ensemble, l'Église constitutionnelle s'adapte bien aux pratiques électorales, avec certes des perturbations dues à la déchristianisation. Comme l'élection des ecclésiastiques, celle des tribunaux de district reste un domaine encore peu étudié. En prenant l'exemple des juges des tribunaux de districts du Nord et du Pas-de-Calais (1790-1792), Hervé Leuwers montre bien là encore une pratique totalement nouvelle par rapport à l'Ancien Régime pour l'accès aux fonctions judiciaires. L'élection des juges de districts et de leurs suppléants se déroule en même temps que celle des administrateurs de district. Elle obéit à des conditions de cens, d'âge - trente ans révolus - et de capacité - avoir exercé pendant cinq ans des fonctions de juge ou d'homme de loi ; les ecclésiastiques sont exclus - ; les 
juges nouveaux sont donc, comme les hommes politiques, des hommes formés par l'Ancien Régime. Les candidats étant peu nombreux, les électeurs sont amenés à élire comme juges des membres de l'assemblée électorale, ce qui représente le quart des juges pour le Pas-de-Calais. H. Leuwers souligne les difficultés pour interpréter les textes et les nombreuses irrégularités constatées dans les procès-verbaux. La majorité des élus sont des avocats ou d'anciens magistrats, locaux ou non, mais à partir de 1792 on assiste à une "ouverture sociale à des hommes de loi sans formation universitaire ".

Le deuxième thème se rapporte à "République, libéralisme et démocratie ». Dans sa communication, Françoise Brunel s'interroge sur «les institutions républicaines: projet démocratique, horizon d'attente et/ou utopie (an II-anV )». En 1948, Albert Soboul écrivait, à propos des Fragments sur les institutions républicaines de Saint-Just: «On a souvent parlé [...] d'utopie de rêve égalitaire ; de république à la spartiate »; F. Brunel reprend le subtil regard de B. Bazcko sur les sens du mot utopie, à savoir un lieu $\mathrm{du}$ bonheur et de la perfection, ou bien une région qui n'existe nulle part? En considérant les différents projets de Saint-Just, de Billaud-Varenne, de Barère ou Babeuf, on trouve cette quête toujours insatisfaite pour établir les meilleures institutions possibles, la constitution la mieux adaptée pour un gouvernement républicain, afin d'émanciper l'humanité toute entière, lutter contre l'ignorance et la misère.

Les travaux de Michel Biard sur les missionnaires de la République (CTHS, 2002) proposent sur la question à la fois une vaste synthèse et une grille de travail tout à fait essentielles. Philippe Bourdin suit ces représentants en mission "entre Rhône et Allier ", en soulignant les difficultés et les périls à gérer la politique locale, puisqu'ils disposent d'une grande autonomie tout en étant étroitement soumis au pouvoir central. Dans cette même optique, Jean-Pierre Jessenne étudie, entre local et national, les pratiques et les liens politiques, du Directoire au Consulat. Il fait référence aux colloques et tables rondes organisés entre 1998 et 2000, insistant sur l'expérience difficile du Directoire avec, selon les régions, des attitudes de rejet ou de collaboration " pour l'exigence des citoyens à être associés aux affaires publiques». Devant toutes ces incertitudes, pouvait on « en sortir par le centre »? La question est posée par Christine le Bozec. Comment définir ce centre... au-delà d'un consensus apparent, d'une instrumentalisation du groupe politique épris de paix et désabusé, incrédule et inquiet face à Bonaparte, clairvoyant et déterminé ? En 1799, Ch. Le Bozec retient des discours les principaux référents politiques: république, unité, indivisibilité nationale tandis que «le gouvernement doit gouverner et les militaires faire la guerre». Et pourtant, "le péril blanc peut ressurgir "; il est vraiment présent et représente selon Bernard Gainot une véritable menace de complot. Reprenant les travaux de J. Godechot sur la contre-révolution, Bernard Gainot renouvelle le concept à travers les différentes stratégies d'écriture et il constate qu'en 1799 contre-révolution et restauration se rapprochent sensiblement, comme en témoigne l'expérience sanglante de la République Napolitaine où les patriotes, - comme dans la Décade - sont fréquemment assimilés à des néo-jacobins (voir les travaux d'A. M. Rao). Le Directoire ne dissipe pas le doute.

11 Ces journées d'études, en ouvrant de nouveaux chantiers prometteurs, se situent bien dans la continuité des travaux de ceux à qui elles ont voulu rendre hommage. 